\title{
L'Annuaire théâtral
}

Revue québécoise d'études théâtrales

\section{Au regard de nouvelles perspectives}

\section{Chantal Hébert}

Numéro 28, automne 2000

Théâtres antillais et guyanais : perspectives actuelles

URI : https://id.erudit.org/iderudit/041433ar

DOI : https://doi.org/10.7202/041433ar

Aller au sommaire du numéro

Éditeur(s)

Centre de recherche en littérature québécoise (CRELIQ) et Société québécoise d'études théâtrales (SQET)

\section{ISSN}

0827-0198 (imprimé)

1923-0893 (numérique)

Découvrir la revue

Citer ce document

Hébert, C. (2000). Au regard de nouvelles perspectives. L'Annuaire théâtral, (28), 5-8. https://doi.org/10.7202/041433ar d'utilisation que vous pouvez consulter en ligne.

https://apropos.erudit.org/fr/usagers/politique-dutilisation/ 


\section{Au REgARD DE NOUVELLES PERSPECTIVES}

À la fin décembre 2000 se terminera mon mandat de directrice de L'Annuaire théâtral. Après quatre ans à la barre de la revue, et à quelques mois d'une année sabbatique, je céderai le gouvernail, ainsi que je l'ai annoncé aux membres de la Société québécoise d'études théâtrales (SQET) en juin 1999. À compter de janvier 2001, L'Annuaire théâtral sera dirigé par Dominique Lafon et aura son port d'attache au Centre de recherche en civilisation canadiennefrançaise (CRCCF) de l'Université d'Ottawa. Dominique Lafon apportera avec elle sa compétence, son ardeur et sa détermination, gages de stabilité et de continuité pour la revue. Le changement d'équipage s'annonce tout à fait prometteur.

Avant de quitter mes fonctions, et au nom de la Société québécoise d'études théâtrales (SQET) et en mon nom personnel, j'aimerais remercier le Centre de recherche en littérature québécoise (CRELIQ) et le Département des littératures de la Faculté des lettres de l'Université Laval pour le soutien qu'ils ont accordé à la revue depuis janvier 1997. De même, je tiens à exprimer à chaque membre du comité de rédaction - Francine Chaîné, Gilbert David, Josette Féral, Shawn Huffman, Dominique Lafon, Hélène Laliberté, Marie-Christine Lesage, Leanore Lieblein, Janusz Przychodzeń, Pascal Riendeau et Rodrigue Villeneuve - le plaisir que j'ai eu à travailler avec eux et elles : merci à vous qui m'avez épaulée par votre constante solidarité et vos précieux conseils. Merci plus particulièrement au rédacteur puis à la rédactrice en chef qu'ont été Gilbert David et MarieChristine Lesage pour leur appui indéfectible. Merci aux membres du conseil scientifique, ainsi qu'à tous les évaluateurs et évaluatrices qui ont accepté, en dépit de leurs occupations toujours plus nombreuses, de lire avec attention les articles qui nous ont été soumis. Enfin, je ne voudrais pas passer sous silence le dynamisme, le professionnalisme et le support de Linda Fortin, coordonnatrice à l'édition de la revue depuis le numéro 21 et idéatrice infatigable. C'est en très large partie à elle et à sa coéquipière, Isabelle Tousignant, responsable de la composition et de l'infographie, toutes deux travaillant de concert avec le comité 
de rédaction, que l'on doit la qualité, remarquable, nous a-t-on dit, que la revue a atteinte, et dont nous avons tout lieu de nous réjouir.

D'autre part, je tiens à signaler que j'ai apprécié la confiance que la Société québécoise d'études théâtrales a placée en moi. J'espère m'être acquittée au mieux de mes responsabilités. Le programme que nous nous étions fixés en janvier 1997 a été respecté (voir "Ici et maintenant ", L'Annuaire théâtral, no 21, printemps 1997). Quatre ans et huit numéros plus tard, parus avec une régularité sans faille, les dossiers annoncés ont tous été traités avec l'" esprit d'ouverture et d'exigence "qui a été le moteur de l'équipe en place, dont le souci était "de s'adapter à l'évolution du phénomène théâtral et de la recherche en théâtre ". D'un numéro à l'autre, nous avons voulu ouvrir des chantiers qui stimuleraient des recherches à venir.

Et cette ouverture, visible tant dans les corpus que dans les modes d'approche, est manifeste une fois de plus dans le présent DOSSIER, "Thêatres antillais et guyanais : perspectives actuelles ". Alvina Ruprecht, maîtresse d'œuvre, en parlera et parlera de ses collaboratrices qui nous font découvrir un imaginaire venu d'ailleurs, celui de la francophonie diraient certains. Pour ma part, je préférerais parler d'un imaginaire métis. L'expression dit mieux, me semble-t-il, la nécessité de sortir d'un certain centralisme et du monologuisme qui risque de lui faire écho. Bien que pouvant être momentanément nécessaire, le monologuisme a tout avantage à déboucher sur le dialogue des cultures pour répondre à l'esprit de l'époque ou d'un monde qui se targue d'être de plus en plus "global ". C'est ce qu'a compris le théâtre québécois qui, après avoir fait de l'affirmation identitaire son cheval de bataille dans les années 1970, s'est mis en quête depuis quelques années déjà de nouveaux horizons, ceux d'un théâtre réceptif au monde. Ce qui est vrai pour le théâtre l'est également pour la recherche théâtrale qui, en s'isolant dans ses préoccupations, risque de s'asphyxier. Ce dossier s'inscrit donc dans la continuité des précédents et atteste de la volonté de L'Annuaire théâtral de créer sur le territoire québécois un "véritable espace de discussion, de connexion entre la pratique et la théorie, un forum réfléchissant le thêâtre d'hier et d'aujourd'hui ", et j'ajouterais d'ici et d'ailleurs, ajustant de ce fait notre vision à de nouvelles perspectives, à d'autres perspectives.

Cette invite au croisement ou au métissage de perspectives, que nous appelions déjà il y a quatre ans en cherchant à faire dialoguer les discours ou recherches théoriques et pratiques, est renforcée par le DOCUMENT, Camille et Justine, texte dramatique inédit de Gerty Dambury, auteure guadeloupéenne, 
née en 1957 à Pointe-à-Pitre, installée à Paris, dont la pièce la plus connue, Lettres indiennes, a été créée à Avignon en 1996, avant d'être reprise à New York en 1997. Camille et Justine, dont le premier jet a été esquissé en 1996, est resté en travail jusqu'au printemps 2000 . C'est cette dernière mouture du texte que nous présentons, dans cette édition, comme " trace concrète $[. .$.$] de la pensée$ créatrice en marche ". Gerty Dambury nous entraîne ici à l'intérieur d'une maison qui devient, à la suite d'une inondation, une barque ou une île à la dérive... Partir, précisera le texte, est inévitable.

La section PRATIQUES \& TRAVAUX regroupe quatre textes dont trois portent sur le monologue. Elle a pour point commun avec le DOSSIER et le DOCUMENT de mettre de l'avant la reconnaissance et l'acceptation de l'altérité, qui peut prendre diverses formes et recouvrir des situations variées, selon les contextes interactionnels ou espaces de communication. Loin de contredire cette condition de la communication, le dialogue avec soi-même la confirme. Dans "Du fondement de la distinction entre monologue et soliloque ", Jean-Jacques Delfour formule l'hypothèse selon laquelle, "dans l'usage du monologue de thêâtre, s'exprime l'esprit du temps " (p. 119). Après avoir cherché à définir au plus près ces notions souvent employées indifféremment, Delfour met de l'avant la porosité des frontières entre les deux types de discours qui, dans un cas comme dans l'autre, sont organisés "autour d'un manque" (p. 127). À ce point de vue philosophique fait écho une perspective pragmatique, celle de Caroline Garand qui revient sur la "Distinction entre monologue et soliloque ", en procédant à "l'étude d'un cas limite, celui de Monologue, de Jean-Pierre Ronfard ". C'est d'un point de vue communicationnel ou de positionnement, c'est-à-dire d'effort de mobilisation d'autrui, qu'elle aborde l'analyse de l'"étrange monologue" (p. 141). Recourant aux cinq modalités de la communication mises au point par Alex Muchielli, Garand dépasse le modèle émetteur-récepteur qui restreint l'analyse à la transmission d'informations pour prendre en compte le contexte et débusquer ce que construit la communication.

À ce texte succède Monologue, extrait du spectacle composite Le cru et le cuit, créé en collaboration par le Nouveau Théâtre Expérimental et Omnibus en novembre 1995. Illustrant la volonté de repousser les frontières en jouant avec les mots sur le mode du monologue, Ronfard interroge le système fondateur de la communication théâtrale basé sur l'échange de paroles alternées ou dialogue, et nous amène à chercher les enjeux de la " situation " de communication au-delà des limites du "dramatique ", comme le propose Garand. Cet inédit de JeanPierre Ronfard, comme celui de Gerty Dambury, fait entrer en résonance les 
perspectives théoriques et pratiques permettant de coconstruire une "représentation "d'un phénomène, d'une problématique, d'une thématique, par le jeu de véritables échanges.

La section se referme sur un texte de Marie-Andrée Brault sur "la question de l'expérimentation " ou du défi aux conventions, qu'elle traite à l'aune de la réception critique de deux spectacles (Treize tableaux et La femme d'intérieur) du Nouveau Théâtre Expérimental (NTE), lieu phare de la liberté expressive et du brassage des formes théâtrales auquel la figure de Jean-Pierre Ronfard, entre autres, est intimement rattachée. S'appuyant sur les travaux de Hans Robert Jauss, Brault rappelle que tout regard posé sur une œuvre est fonction d'un horizon d'attente, et qu'il en a été de même de celui qu'a jeté la critique sur les explorations menées par le NTE à la fin des années 1970, expliquant par là la réception accueillante de l'œuvre " autre ".

Nos abonnés et nos abonnées se souviendront peut-être que, en raison de l'ampleur du numéro spécial marquant le quinzième anniversaire de la revue $\left(n^{\circ} 27\right)$ au printemps 2000 , nous avions reporté la publication de la section PRATIQUES \& TRAVAUX à cet automne. Voilà avec la parution de celle-ci réponse à nos promesses et à certaines attentes... Enfin, le numéro se referme, lui, sur les NOTES DE LECTURE habituelles, suivies de la REVUE DES REVUES DE LANGUE FRANÇAISE qui revient tous les automnes.

Là-dessus je souhaite "Bon vent! " à L'Annuaire théâtral et à Dominique Lafon, ma successeure. La revue, qui sera en bonnes mains, a un bel avenir en perspective. 